\title{
Efficient solving of engineering design problems by an interior point 3-D filter line search method
}

\author{
M. Fernanda P. Costa* and Edite M.G.P. Fernandes ${ }^{\dagger}$ \\ * Departamento de Matemática para a Ciência e Tecnologia \\ ${ }^{\dagger}$ Departamento de Produção e Sistemas \\ Universidade do Minho, 4710-057 Braga, Portugal
}

\begin{abstract}
Here we present an interior point three-D filter line search method for solving optimization problems. Each entry in the filter includes the feasibility measure, the centrality measure and the barrier objective function value. The algorithm is tested with benchmark engineering design problems, and a comparison with other published results are also included.
\end{abstract}

Keywords: nonlinear programming, interior point method, filter method, engineering design

PACS: $02.60 . \mathrm{Pn}$

\section{INTRODUCTION}

The optimization problems herein addressed are of the form:

$$
\begin{array}{cl}
\min _{x \in \mathbb{R}^{n}} & F(x) \\
\text { subject to } & h(x) \geq 0
\end{array}
$$

where $h_{i}: \mathbb{R}^{n} \rightarrow \mathbb{R}$ for $i=1, \ldots, m$ and $F: \mathbb{R}^{n} \rightarrow \mathbb{R}$ are nonlinear and twice continuously differentiable functions.

In this paper, we use the filter technique of Fletcher and Leyffer [1] to globalize an interior point method for solving problems like (1). The filter technique uses the concept of nondominance present in the multi-objective optimization to build a filter that is able to reject poor trial iterates and enforce global convergence from arbitrary starting points. Filter techniques have already been incorporated into interior point methods. In Ulbrich, Ulbrich and Vicente [2], a filter trust-region strategy based on two components is proposed. In [3, 4], a filter line search strategy that relies on two components is used. These components are the barrier objective function and the constraints violation. A nonmonotone line search filter approach is presented in [5]. The filter therein proposed has three components that measure feasibility, centrality and optimality. The optimality measure is based on the norm of the gradient of the Lagrangian function associated to the barrier problem.

This paper aims to present a 3-D filter line search approach that uses, together with the well-known feasibility and centrality measures of a typical primal-dual interior point method, the barrier objective function to measure the optimality level of trial iterates. Performance assessment of the proposed algorithm is carried out with a set of benchmark engineering design problems.

\section{INTERIOR POINT 3-D FILTER LINE SEARCH METHOD}

This section briefly describes a primal-dual interior point method for solving (1). Adding nonnegative slack variables $w$ to transform the inequality constraints into equality constraints, and incorporating the constraints $w \geq 0$ in logarithmic barrier terms in the objective function, (1) is transformed into the barrier problem

$$
\begin{array}{cl}
\min _{x \in \mathbb{R}^{n}, w \in \mathbb{R}^{m}} & \varphi_{\mu}(x, w) \\
\text { subject to } & h(x)-w=0,
\end{array}
$$


where $\varphi_{\mu}(x, w)=F(x)-\mu \sum_{i=1}^{m} \log \left(w_{i}\right)$ is the barrier function and $\mu$ is a positive barrier parameter. The solution to the problem (2) satisfies the following primal-dual system

$$
\begin{aligned}
\nabla F(x)-A^{T} y & =0 \\
-\mu e+W Y e & =0 \\
h(x)-w & =0
\end{aligned}
$$

where $y$ is the dual variable, $\nabla F$ is the gradient vector of $F, A$ is the Jacobian matrix of the constraints $h, W=\operatorname{diag}\left(w_{i}\right)$ and $Y=\operatorname{diag}\left(y_{i}\right)$ are diagonal matrices of order $m$, and $e$ is an $m$ vector of all ones. Applying the Newton's method to solve (3), a reduced KKT system is obtained for $(\Delta x, \Delta y)$

$$
\left[\begin{array}{ll}
-H(x, y) & A^{T} \\
A & W Y^{-1}
\end{array}\right]\left[\begin{array}{l}
\Delta x \\
\Delta y
\end{array}\right]=\left[\begin{array}{l}
\nabla F(x)-A^{T} y \\
\mu Y^{-1} e-h(x)
\end{array}\right],
$$

where $H(x, y)=\nabla^{2} F(x)-\sum_{i=1}^{m} y_{i} \nabla^{2} h_{i}(x)$ represents the Hessian of the Lagrangian function associated to (2). Then $\Delta w=W Y^{-1}\left(\mu W^{-1} e-y-\Delta y\right)$. For simplicity, we use the following notation:

$$
u=(x, w, y), u^{1}=(x, w), u^{2}=(w, y), \Delta=(\Delta x, \Delta w, \Delta y), \Delta^{1}=(\Delta x, \Delta w), \Delta^{2}=(\Delta w, \Delta y) .
$$

From an initial point $x_{0}, w_{0}>0$ and $y_{0}>0$ the algorithm proceeds iteratively choosing a step size $\alpha_{k}$, at iteration $k$, and defining a new approximation by $u_{k+1}=u_{k}+\alpha_{k} \Delta_{k}$. The step size $\alpha_{k}$ should guarantee the nonnegativity of the slack and dual variables. The procedure that decides which trial step size is accepted is a 3-D filter line search method. A filter is a set $\bar{F}_{k}$ that contains values of $\theta_{f}, \theta_{c}$ and $\varphi_{\mu}$, that are prohibited for a trial iterate to be acceptable in iteration $k$. These three components of each entry in the filter measure feasibility, centrality and optimality, respectively, and they are defined as follows:

$$
\theta_{f}\left(u^{1}\right)=\|w-h(x)\|_{2}, \theta_{c}\left(u^{2}\right)=\left\|\mu W^{-1} e-y\right\|_{2} \text { and } \varphi_{\mu}\left(u^{1}\right) \text { is the barrier function. }
$$

The algorithm also implements a backtracking line search procedure, defining a decreasing sequence of step sizes $\alpha_{k, l} \in\left(0, \alpha_{k}^{\max }\right](l=0,1, \ldots)$, with $\lim _{l} \alpha_{k, l}=0$, until an acceptance criterion is satisfied. Here, $l$ denotes the iteration counter for the inner loop. $\alpha_{k}^{\max }$ is the longest step size that can be taken along $\Delta_{k}$ before the nonnegativity conditions $w_{k} \geq 0$ and $y_{k} \geq 0$ being violated, with an upper bound of 1. Using this 3-D filter method, the trial iterate $u_{k}\left(\alpha_{k, l}\right)=u_{k}+\alpha_{k, l} \Delta_{k}$ is accepted by the filter if it leads to sufficient progress in one of the three measures, when compared to the current iterate:

$$
\begin{aligned}
\theta_{f}\left(u_{k}^{1}\left(\alpha_{k, l}\right)\right) \leq & \left(1-\gamma_{\theta_{f}}\right) \theta_{f}\left(u_{k}^{1}\right) \text { or } \theta_{c}\left(u_{k}^{2}\left(\alpha_{k, l}\right)\right) \leq\left(1-\gamma_{\theta_{c}}\right) \theta_{c}\left(u_{k}^{2}\right) \\
& \text { or } \varphi_{\mu}\left(u_{k}^{1}\left(\alpha_{k, l}\right)\right) \leq \varphi_{\mu}\left(u_{k}^{1}\right)-\gamma_{\varphi} \theta_{f}\left(u_{k}^{1}\right)
\end{aligned}
$$

where $\gamma_{\theta_{f}}, \gamma_{\theta_{c}}, \gamma_{\varphi} \in(0,1)$ are fixed constants. However, to prevent convergence to a feasible but nonoptimal point, and whenever for the trial step size $\alpha_{k, l}$, the following switching conditions

$$
m_{k}\left(\alpha_{k, l}\right)<0 \text { and }\left[-m_{k}\left(\alpha_{k, l}\right)\right]^{s_{o}}\left[\alpha_{k, l}\right]^{1-s_{o}}>\delta\left[\theta_{f}\left(u_{k}^{1}\right)\right]^{s_{f}} \text { and }\left[-m_{k}\left(\alpha_{k, l}\right)\right]^{s_{o}}\left[\alpha_{k, l}\right]^{1-s_{o}}>\delta\left[\theta_{c}\left(u_{k}^{2}\right)\right]^{s_{c}}
$$

hold, with fixed constants $\delta>0, s_{f}>1, s_{c}>1, s_{o} \geq 1$, where $m_{k}(\alpha)=\alpha \nabla \varphi_{\mu}\left(u_{k}^{1}\right)^{T} \Delta_{k}^{1}$, then the trial iterate must satisfy the Armijo condition

$$
\varphi_{\mu}\left(u_{k}^{1}\left(\alpha_{k, l}\right)\right) \leq \varphi_{\mu}\left(u_{k}^{1}\right)+\eta_{o} m_{k}\left(\alpha_{k, l}\right),
$$

instead of (5), to be acceptable. Here, $\eta_{o} \in(0,0.5)$ is a constant. At the beginning of the iterative process, the filter is initialized to $\bar{F}_{0} \subseteq\left\{\left(\theta_{f}, \theta_{c}, \varphi_{\mu}\right) \in \mathbb{R}^{3}: \theta_{f} \geq \theta_{f}^{\max }, \theta_{c} \geq \theta_{c}^{\max }, \varphi_{\mu} \geq \varphi_{\mu}^{\max }\right\}$ for some positive constants $\theta_{f}^{\max }, \theta_{c}^{\max }$ and $\varphi_{\mu}^{\max }$. Whenever the accepted step size satisfies (5), the filter is updated by

$$
\bar{F}_{k+1}=\bar{F}_{k} \cup\left\{\left(\theta_{f}, \theta_{c}, \varphi_{\mu}\right) \in \mathbb{R}^{3}: \theta_{f} \geq\left(1-\gamma_{\theta_{f}}\right) \theta_{f}\left(u_{k}^{1}\right) \text { and } \theta_{c} \geq\left(1-\gamma_{\theta_{c}}\right) \theta_{c}\left(u_{k}^{2}\right) \text { and } \varphi_{\mu} \geq \varphi_{\mu}\left(u_{k}^{1}\right)-\gamma_{\varphi} \theta_{f}\left(u_{k}^{1}\right)\right\},
$$

and it remains unchanged if for the accepted step size, (6) and (7) hold. Finally, when the trial step size $\alpha_{k, l}$ becomes too small, the algorithm reverts to a restoration phase that aims to find a new iterate $u_{k+1}$ that is acceptable to the current filter, for which (5) holds, by decreasing either the feasibility measure $\frac{1}{2}\|w-h(x)\|_{2}^{2}$ or the centrality measure $\frac{1}{2}\left\|\mu W^{-1} e-y\right\|_{2}^{2}$. The reader is referred to [6] for details. 


\section{NUMERICAL EXPERIMENTS WITH ENGINEERING DESIGN PROBLEMS}

Problems of practical interest are important for assessing the effectiveness of any algorithm. Thus, to evaluate the performance of our interior point 3-D filter line search method a set of 7 benchmark engineering problems is used. Comparisons with other published results are also included. The parameters were defined as follows (as in [4]): $\theta_{f}^{\max }=10^{4} \max \left\{1, \theta_{f}\left(u_{0}^{1}\right)\right\}, \theta_{c}^{\max }=10^{4} \max \left\{1, \theta_{c}\left(u_{0}^{2}\right)\right\}, \varphi_{\mu}^{\max }=10^{4} \max \left\{1, \varphi_{\mu}\left(u_{0}^{1}\right)\right\}, \gamma_{\theta_{f}}=\gamma_{\theta_{c}}=\gamma_{\varphi}=10^{-5}, \delta=1$, $s_{f}=s_{c}=1.1, s_{o}=2.3$ and $\eta_{o}=10^{-4}$.

This algorithm is a quasi-Newton based method in the sense that a symmetric positive definite quasi-Newton BFGS approximation, $B_{k}$, is used to approximate the Hessian of the Lagrangian $H$, at each iteration $k$. Initial approximations to the variables are as follows: (i) $x_{0}$ is taken as published in [10] and $y_{0}$ is set to 1 ; or, a better approximation, say $\widetilde{x}_{0}$, as well as $y_{0}$, are computed by solving a simplified reduced KKT (see (4)) based on the published $x_{0}$; (ii) the initial matrix $B_{0}$ is a positive definite modification of $\nabla^{2} F\left(x_{0}\right)$; or, $B_{0}$ is set to the identity matrix, due to numerical difficulties; (iii) $w_{0}=\max \left\{\left|h\left(x_{0}\right)\right|, 0.01\right\}$. The $\mu_{k}$ values are generated with a formula similar to the one used in [4], in order to guarantee a sequence of decreasing positive values. The main characteristics of the chosen engineering problems are as follows. They all have bound and inequality constraints. These problems are fully described in the below cited references.

(1) Design of a welded beam [7, 8, 9, 10,11, 12]. In this problem, the cost of a welded beam is minimized, subject to constraints on the shear stress, bending stress in the beam, buckling load on the bar, end deflection of the beam, and side constraints. The problem has 4 design variables and 7 inequality constraints.

(2) Design of a heat exchanger [7, 9, 10]. This problem minimizes the sum of the heat transfer areas of three exchangers, and has 8 design variables and 6 inequality constraints.

(3) Design of a speed reducer $[10,12]$. The objective in this problem is to minimize the total weight of a speed reducer, subject to constraints on bending stress of the gear teeth, surface stress, transverse deflections of the shafts and stresses in the shafts. It has 7 design variables and 11 inequality constraints.

(4) Design of a tension/compression spring $[8,10,11,12]$. This problem minimizes the weight of a tension/compression spring, subject to constraints on the minimum deflection, shear stress, surge frequency, limits on outside diameter and on design variables. The problem has 3 design variables and 4 inequality constraints.

(5) Design of three-bar truss [12]. This problem minimizes the volume of a 3-bar truss structure, subject to stress constraints. The problem has 2 design variables that represent cross-sectional areas of two bars (the third bar is equal to the first bar), and 3 inequality constraints.

(6) Design of a tubular column [10]. The objective is to minimize the total cost of the material and construction of a tubular column. The problem has 2 design variables and 2 inequality constraints.

(7) Design of a pressure vessel $[8,9,11]$. In this problem, the total cost of the material, forming and welding of a cylindrical pressure vessel is minimized. The problem has 4 design variables and 4 inequality constraints.

The results reported in Table 1 include the number of iterations $\left(N_{i t}\right)$, the number of objective function evaluations $\left(N_{f e}\right)$ and the solution found $\left(F^{*}\right)$ with the tolerance $10^{-4}$ in the stopping criteria. These are similar to the ones in [4], and are based on relative measures, using equations (3). The results $N_{i t}$ and $N_{f e}$ that are from stochastic methods correspond to average values obtained over a pre-defined number of runs. The $F^{*}$ value is the best function value out of the specified runs. The results of the first two rows correspond to the method herein proposed. The third and fourth rows show the results obtained when the third component of the filter is based on the norm of the gradient of the Lagrangian function $\left(\left\|\nabla_{x} L\right\|\right)$ of problem (2). The remaining rows list results obtained by other classes of methods. In the table, "-" means unavailable information, and values marked with (+) correspond to the weight of the 3-bar truss structure instead of the volume. Details concerning the methods whose results are reported in the table are as follow: (a) stochastic population-based method, and best solution found over 50 runs with a population of 80 points; (b) stochastic point-to-point search method, and best solution found over 30 runs (50 for beam problem); (c) stochastic population-based method, and best solution found over 20 runs with a population of 20 points; (d) the algorithm stops when change in $F$ in consecutive iterations is less than $0.01 \%$, and constraints are satisfied with a tolerance of $0.01 \%$ of the initial values of the constraints; (e) stochastic population-based method, and best solution found over 100 runs with a population of 20 points; (f) stochastic population-based method, and best solution found over 50 runs with a population of $10 n$ points ( $n$ is the number of variables); $(\mathrm{g}$ ) stochastic population-based method, and best solution found over 30 runs with a population of 100 points; (h) results reported in [10] from other deterministic methods. We may conclude that our algorithm is effective in reaching the best solution known in the literature. It is a promising and efficient strategy for solving engineering problems. 
TABLE 1. Comparison with other classes of methods.

\begin{tabular}{rrrrrrrrr} 
& & \multicolumn{1}{c}{ beam } & \multicolumn{1}{c}{ heat } & speed & \multicolumn{1}{c}{ spring } & truss & tubular & vessel \\
\hline$\phi_{\mu}$ & $N_{i t} / N_{f e}$ & $36 / 195$ & $43 / 67$ & $126 / 162$ & $42 / 170$ & $24 / 25$ & $36 / 37$ & $31 / 40$ \\
& $F^{*}$ & 2.38097 & 7049.25 & 2994.35 & $1.2665 \mathrm{e}-2$ & 263.8958 & 26.53132 & 5885.33 \\
$\left\|\nabla_{x} L\right\|$ & $N_{i t} / N_{f e}$ & $70 / 71$ & $44 / 45$ & $77 / 78$ & $26 / 27$ & $12 / 13$ & $36 / 37$ & $24 / 25$ \\
& $F^{*}$ & 2.38097 & 7049.25 & 2994.35 & $1.2665 \mathrm{e}-2$ & 263.8958 & 26.53132 & 5885.33 \\
[7] (a) & $N_{i t} / N_{f e}$ & $4000 / 40080$ & $4000 / 320080$ & - & - & - & - & - \\
& $F^{*}$ & 2.38119 & 7060.221 & - & - & - & - & - \\
[8] (b) & $N_{i t} / N_{f e}$ & $-/ 56243$ & - & - & $-/ 49531$ & - & - & $-/ 108883$ \\
& $F^{*}$ & 2.38107 & - & - & $1.26653 \mathrm{e}-2$ & - & - & 5868.77 \\
[9] (c) & $N_{i t} / N_{f e}$ & $-/ 110000$ & $-/ 150000$ & - & - & - & - & - \\
& $F^{*}$ & 2.38 & 7057.274 & - & - & - & - & 7198.433 \\
[10] (d) & $N_{i t} / N_{f e}$ & $139 /-$ & $1963 /-$ & $302 /-$ & $225 /-$ & $75 /-$ & $53 /-$ & - \\
& $F^{*}$ & 2.0182 & 7288.8 & 3007.8 & $1.2650 \mathrm{e}-2$ & $2.6507(+)$ & 25.5316 & - \\
[11] (e) & $N_{i t} / N_{f e}$ & $5000 /-$ & - & - & $5000 /-$ & - & - & $5000 /-$ \\
& $F^{*}$ & 1.76558 & - & - & $1.2816 \mathrm{e}-2$ & - & - & 6154.70 \\
[12] (f) & $N_{i t} / N_{f e}$ & $1000 / 33095$ & - & $1000 / 54456$ & $1000 / 25167$ & $1000 / 17610$ & - & - \\
& $F^{*}$ & 2.38543 & - & 2994.74 & $1.2669 \mathrm{e}-2$ & 263.8958 & - & - \\
[13] (g) & $N_{i t} / N_{f e}$ & $-/ 30000$ & - & $-/ 40000$ & $-/ 24000$ & $-/ 15000$ & - & - \\
& $F^{*}$ & 2.380957 & - & 2994.499 & $1.26652 \mathrm{e}-2$ & 263.8958 & - & - \\
(h) & $F^{*}$ & 2.386 & 7049 & 2985.2 & $1.2833 \mathrm{e}-2$ & $2.6335(+)$ & 26.5323 & 6154.70 \\
\hline
\end{tabular}

\section{CONCLUSIONS AND FUTURE WORK}

We presented an efficient interior point 3-D filter line search method for solving practical engineering design problems. Each entry in the filter has three components measuring feasibility, centrality and optimality, being the latter based on the barrier function value. Comparing with other deterministic and stochastic algorithms, our proposal is by far the most efficient and effective. Other interesting and complex engineering problems, such as larger truss sizing and configuration optimization problems will be addressed in the future.

\section{REFERENCES}

1. R. Fletcher and S. Leyffer, Nonlinear programming without a penalty function, Mathematical Programming 91, 2002, 239-269.

2. M. Ulbrich, M. S. Ulbrich, and L.N. Vicente, A globally convergent primal-dual interior-point filter method for nonlinear programming, Mathematical Programming, 100, 2004, 379-410.

3. A. Wächter and L.T. Biegler, Line search filter methods for nonlinear programming: motivation and global convergence, SIAM Journal on Optimization, 16, 2005, 1-31.

4. A. Wächter and L.T. Biegler, On the implementation of an interior-point filter line-search algorithm for large-scale nonlinear programming, Mathematical Programming, 106, 2006, 25-57.

5. M.F.P. Costa, and E.M.G.P. Fernandes, Practical implementation of an interior point nonmonotone line search filter method, International Journal of Computer Mathematics, 85, 2008, 397-409.

6. M.F.P. Costa, and E.M.G.P. Fernandes, A three-D filter line search method within an interior point framework, Proceedings of 2008 ICMMSE, ISBN: 978-84-612-1982-7, 2008, 173-187.

7. K. Deb, An efficient constraint handling method for genetic algorithms, Computer Methods in Applied Mechanics and Engineering, 186, 2000, 311-338.

8. A.-R. Hedar and M. Fukushima, Derivative-free filter simulated annealing method for constrained continuous global optimization, Journal of Global Optimization, 35, 2006, 521-549.

9. K. S. Lee and Z. W. Geem, A new meta-heuristic algorithm for continuous engineering optimization: harmony search theory and practice, Computer Methods in Applied Mechanics and Engineering, 194, 2005, 3902-3933.

10. T.-C. Liu, Developing a fuzzy proportional-derivative controller optimization engine for engineering optimization problems, $\mathrm{PhD}$ thesis, August 2006.

11. K. E. Parsopoulos and M. N. Vrahatis, Unified particle swarm optimization for solving constrained engineering optimization problems, Lecture Notes in Computer Science, 3612, 2005, 582-591.

12. T. Ray and K. M. Liew, Society and Civilization: an optimization algorithm based on the simulation of social behavior, IEEE Transactions on Evolutionary Computation, 7, 2003, 386-396.

13. Y. Wang, Z. Cai, Y. Zhou and Z. Fan, Constrained optimization based on hybrid evolutionary algorithm and adaptive constraint-handling technique, Structural Multidisciplinary Optimization, DOI 10.1007/s00158-008-0238-3. 